\title{
Mallorca y sus dinámicas metropolitanas: proximidad y movilidad cotidiana en una isla-ciudad
}

\author{
Jaume Mateu. Universidad de las Islas Baleares, Islas Baleares, Espańa. \\ Joana Maria Seguí. Universidad de las Islas Baleares, Islas Baleares, España. \\ Maurici Ruiz. Universidad de las Islas Baleares, Islas Baleares, Espańa.
}

RESUMEN | Los viajes de proximidad son los desplazamientos más indicados para reducir la movilidad en vehículo privado y favorecer la equidad social y la sostenibilidad ambiental en los núcleos urbanos. Desplazamientos de este tipo son más característicos de las ciudades tradicionales, compactas y complejas, pero han sido sustituidos por traslados de larga distancia en el proceso de dispersión urbana acentuado en las últimas décadas. La isla de Mallorca no ha sido ajena a esta dinámica, pues se ha consolidado como un espacio metropolitano en el que conviven los núcleos urbanos tradicionales con nuevos espacios residenciales dispersos. En este contexto se examinan los desplazamientos de proximidad a partir de un análisis clúster según perfiles sociodemográficos, modos y motivos de desplazamiento. El resultado indica que los factores socioeconómicos y la cuestión de género son aspectos relevantes a la hora de comprender los desplazamientos cotidianos de proximidad.

PALABRAS ClAVE $\mid$ movilidad, desigualdad social, estructura urbana.

ABSTRACT | Proximity trips are the ideal kind of trips to reduce private vehicle mobility, and promote social equity and environmental sustainability in urban centers. These kinds of trips are more characteristic of traditional, compact and complex cities, but have been replaced by long distance trips due to the development of urban sprawl in the recent decades. Mallorca Island has not been exempt to this dynamic, as it has established itself as a metropolitan space in which traditional urban cores coexist with scattered new residential spaces. In this context, movements of proximity are analyzed and classified, based on a cluster analysis according to socio-demographic profiles, modes and motives of journey. The results suggest that socioeconomic, equity and gender aspects are key issues to understanding daily proximity trips.

KEYwORDs | mobility, social inequality, urban structure. 


\section{Introducción. Objetivos y planteamiento}

La dialéctica entre la forma urbana y las conductas de movilidad inducidas desde los años sesenta por la apuesta por el automóvil como herramienta de transporte, han sido profundamente analizadas por una gran diversidad de autores. Transporte y ciudad son dos caras de una relación compleja: el transporte condiciona la forma urbana, y la forma urbana condiciona el transporte (Miralles-Guasch, 2002). Desde que Lewis Mumford vaticinara una explosión urbana derivada de la popularización del automóvil, diversos análisis apuntan a que el modelo urbano disperso, acompañado de una red de autopistas y autovías y de un incremento constante de consumo de territorio, se ha ido extendiendo hacia todos los rincones del planeta. La expansión urbana del modelo disperso, importada en Europa desde Norteamérica, incide no solo en una pérdida de calidad paisajística y ambiental, sino también en un cambio del modelo urbano compacto, más propio de las ciudades europeas (y sobre todo mediterráneas), por otro más caracterizado por la separación de actividades y la aparición de territorios monofuncionales que conducen a una mayor necesidad de desplazamientos. Hemos construido espacios para residencia, trabajo y ocio muy separados entre sí, pero unidos por complejas y extensas redes de transporte. Los desplazamientos diarios se hacen imprescindibles y, a veces, a distancias realmente considerables.

Esos nuevos modelos urbanos polifuncionales han sido analizados en profundidad en Europa y en España, a través de los conceptos de ciudad difusa (Indovina, 2007; Nel·lo, 2012), producción residencial de baja densidad (Muńoz, 2005) o los vinculados al análisis del urban sprawl y sus efectos en términos ambientales y territoriales (Esteban, 2006; Ludlow, 2006). En muchos trabajos aparece también desarrollada la relación entre modelo urbano y movilidad (García-Palomares, 2010; Travisi, Camagni \& Nijkamp, 2010). Estos autores coinciden en que el modelo urbano disperso genera más desplazamientos motorizados en vehículo privado, debido a la menor capacidad de acceder al transporte colectivo que conlleva la urbanización de baja densidad (De Vos \& Witlox, 2013). Ello se traduce en mayores distancias, mayores costes y menor accesibilidad en transporte público, con diferencias importantes de servicio e impactos en la equidad social (Currie, 2010).

Como contrapunto a este modelo de dispersión urbana aparece la ciudad tradicional, compacta y compleja, con menos necesidad de movimientos y con una mayor accesibilidad a todos los segmentos de población gracias a la mayor cobertura en transporte colectivo. Se produce igualmente una mayor facilidad para los viajes no motorizados, debido a la mezcla de usos del suelo y de actividades que aproxima entre sí a las diferentes zonas de la ciudad, haciéndolas más accesibles. Por ello, entre las medidas que favorecen la sostenibilidad de los planes de movilidad urbana se sitúan las que vinculan las herramientas de planificación de la ciudad y las de la movilidad (Wefering, Rupprecht, Bührmann, Böhler-Baedeker \& Rupprecht Consult, 2013), de manera de favorecer tejidos urbanos más compactos y menos generadores de aquellos desplazamientos consecuentes a la dispersión de usos. Los viajes de proximidad, los que se producen en el entorno cercano, constituyen así un buen indicador de la sostenibilidad urbana, ya que reducen los impactos ambientales y sociales derivados de la ciudad dispersa y de sus dinámicas de movilidad. 
En este sentido, algunos autores plantean un análisis de los viajes de corto alcance y en modos no motorizados como muestra de los procesos de cercanía urbana. La importancia de este tipo de desplazamientos radica en constituir una buena muestra de la movilidad cotidiana de la población que transcurre "sobre tres ejes principales de reflexión: la cuestión del medio ambiente, la importancia de la walkability ${ }^{1}$ y el uso del tiempo social” (Marquet \& Miralles-Guasch, 2013, p. 101). Estos vectores representan una buena aproximación a las características e importancia de los desplazamientos de proximidad para favorecer precisamente modelos urbanos más sostenibles, de compacidad y mixticidad en los usos del suelo. En términos ambientales, la reducción de desplazamientos y el mayor uso de modos no motorizados y colectivos rebajan el impacto ambiental de los transportes en las ciudades. Los viajes de proximidad pueden aumentar a partir de una mejora de los espacios urbanos que fomente los desplazamientos a pie y en bicicleta. A su vez, el peso de los desplazamientos de proximidad y sus características constituyen un buen reflejo del modelo urbano existente en un espacio concreto.

Varios son los autores que también han analizado la evolución de las relaciones espaciotemporales y sus consecuencias en las dinámicas de movilidad. Buliung (2011) examina los efectos espaciales de los cambios tecnológicos en la vida cotidiana, e identifica la fragmentación de las actividades como uno de los factores responsables del auge de la hipermovilidad. Tal y como apuntan también Ben Elia, Alexander, Hubers y Ettema (2014), esta fragmentación es la que ha producido un cambio en nuestros hábitos diarios, de manera que desde una situación en la que el tiempo y el espacio dedicados a trabajo, ocio y vida familiar estaban claramente delimitados, estamos evolucionando hacia una nueva situación en la que tanto tiempo como espacio se fragmentan y se intercambian, gracias a las posibilidades que ofrecen las nuevas tecnologías, hecho que se traduce también en un incremento de los desplazamientos cotidianos.

A partir de esta línea de investigación y de las reflexiones en torno a ella, planteamos un análisis de los viajes de proximidad en la isla de Mallorca, en nuestro caso entendidos como los viajes que se producen en una misma zona y con una duración máxima de diez minutos, independientemente del modo y motivo de su realización. Así, partimos de los datos de la última gran encuesta de movilidad realizada en día laborable para el conjunto de Mallorca, en 2009, para analizar los motivos, los perfiles sociodemográficos y los modos utilizados en los viajes de proximidad, así como su distribución horaria y su relación con la estructura del sistema urbano insular.

\section{Ámbito de estudio}

Ubicada en el Mediterráneo occidental, la isla de Mallorca suele ser reconocida e identificada por su perfil turístico. Efectivamente, el desarrollo del turismo en las Baleares, y en concreto del turismo de masas, ha supuesto su transformación intensa

1 El término walkability hace referencia al atractivo de los espacios urbanos para su uso por parte de los peatones. Algunos autores lo han traducido al castellano con el vocablo "caminabilidad". Mantenemos el término en inglés por ser la cita literal del trabajo en referencia. 
a lo largo de los últimos cincuenta años. El número anual de turistas que visitan las Baleares alcanzó los 13,5 millones en 2014, cifra muy relevante para una población de 1,1 millones de habitantes. El proceso de apertura económica y la construcción de una gran infraestructura de alojamiento en su litoral para acoger la creciente demanda del turismo de sol y playa han transformado la Isla. El consecuente incremento turístico y demográfico, atraído por la demanda de mano de obra, ha contemplado un desarrollo urbano muy relevante, con un avance de los usos urbanos en el conjunto del territorio insular, una pérdida de valor de la actividad agraria y una terciarización generalizada de la economía. Ya en la década de los setenta se describía la Isla como un único sistema urbano interconectado (Quintana, 1979), caracterizado por la macrocefalia de la ciudad de Palma. Fruto de este proceso de "metropolitanización”, en los últimos decenios se ha ido mitigando la separación urbana entre capital y resto del territorio, para conformar un conjunto mucho más integrado y mucho más interdependiente (Mestre, 2014).

El proceso de construcción de un sistema metropolitano en toda la isla de Mallorca se inició en los ańos cincuenta del siglo xx, en paralelo al inicio del turismo de masas, una actividad de crecimiento explosivo que conllevó la urbanización del litoral de la isla hasta ese momento prácticamente intocado. A la vez, los pueblos de interior fueron perdiendo su carácter rural para convertirse paulatinamente en espacios de residencia de los trabajadores del sector servicios, lo que culminó en la pérdida de actividad agrícola y la integración de todo el territorio insular en un único sistema metropolitano interconectado.

Formalmente este sistema se caracteriza por la existencia de tres tejidos urbanos principales (Mestre, 2014): los núcleos urbanos tradicionales (la ciudad de Palma y los núcleos urbanos de los pueblos de interior); las zonas turísticas y urbanizaciones litorales; y los más recientes tejidos urbanos dispersos, que adoptan la forma de urbanizaciones con predominio de viviendas unifamiliares o de nuevas construcciones en espacios rurales.

Como acelerador del proceso de urbanización cabe mencionar la reducción de las distancias que conllevó la ampliación de la red de carreteras y autopistas iniciada en la década de los noventa y, consecuentemente, el incremento de la demanda residencial hacia el interior insular. Este movimiento centrífugo emitido desde la ciudad de Palma aumentó de forma considerable la movilidad laboral atribuida a la separación de espacios residenciales y centros de trabajo, debido a que muchos residentes en Palma se trasladaron a vivir a los pueblos de interior, manteniendo la ciudad como lugar de trabajo. De este modo, la Isla ha ido convirtiéndose paulatinamente en un gran espacio residencial y turístico. Los desplazamientos pivotan sobre el tráfico motorizado privado, que incrementa la congestión de las vías de acceso y de las que circunvalan la ciudad de Palma, en proceso de expansión permanente (Seguí Pons, 2009). El parque automovilístico de Mallorca es elevado. En 2012 se registraban 811 vehículos por cada 1000 habitantes, una de las tasas más altas de España, a pesar del descenso de los últimos años motivado por la crisis económica. En paralelo, el transporte colectivo ha tenido poco recorrido. Básicamente centrado en el sistema de transporte público de Palma, el único transporte urbano insular está constituido por una línea de metro, unas pocas líneas ferroviarias y el sistema 
de buses interurbanos, todos ellos mejorados en las dos últimas décadas. Fruto de esas mejoras se han incrementado los pasajeros en transporte público y colectivo.

Esta situación se refleja claramente en la caracterización de los desplazamientos cotidianos en la Isla. Los datos de movilidad en un día laborable en Mallorca, procedentes de la encuesta de movilidad del Consorcio de Transportes de Mallorca en 2009, muestran cómo el transporte en vehículo privado y motorizado es mayoritario, con más del 50\% del total de los desplazamientos en día laborable, casi todos ellos en automóvil. Por el contrario, los viajes en modos no motorizados alcanzan solamente el $40 \%$ de los movimientos, siendo el modo "a pie" el más destacado. Únicamente el $7,2 \%$ de los desplazamientos corresponde al transporte público colectivo, con preponderancia del transporte público urbano de Palma (5\%) y un peso mucho menor del resto de modos (transporte interurbano, metro y tren).

\section{FIGURA I | Ámbito de estudio}

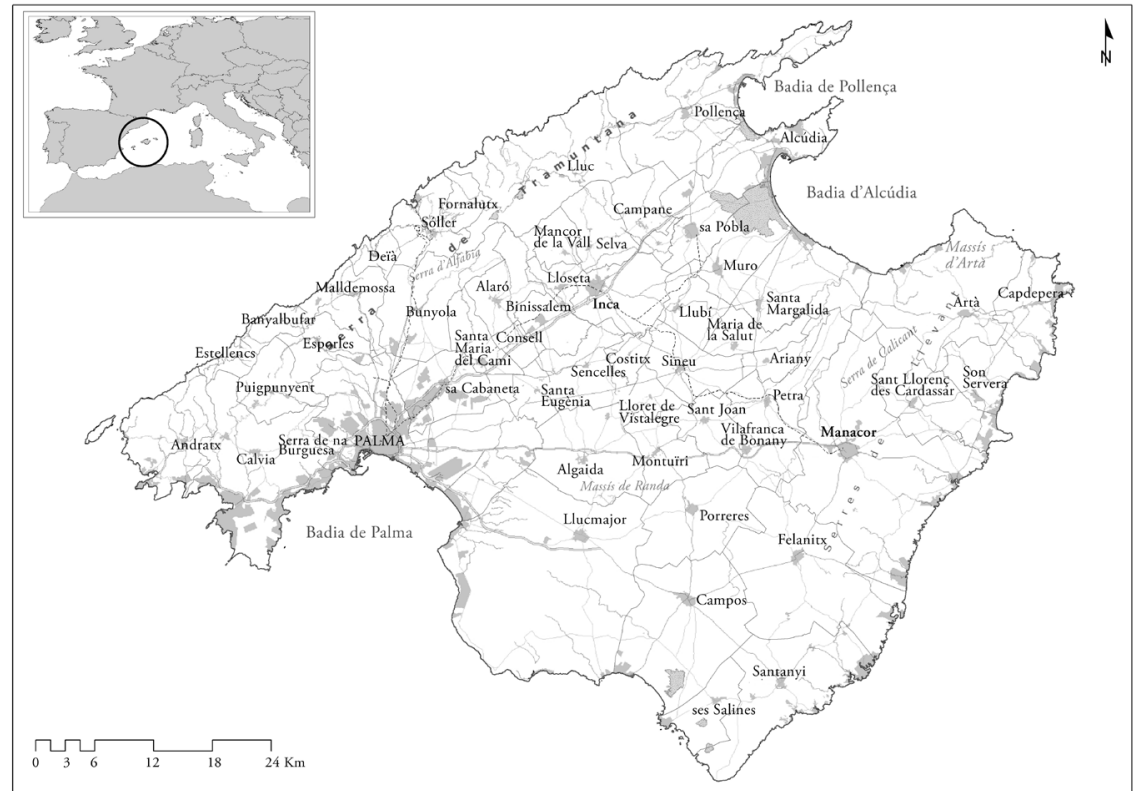

FUENTE MAPA TOPOGRÁFICO BALEAR (SERVEIS D'INFORMACIÓ TERRITORIAL DE LES ILLES BALEARS [SITIBSA], 20I 5)

La estructura territorial de Mallorca (figura 1), en la que Palma y su área metropolitana concentran cerca del $50 \%$ de la población de la Isla, tiene su correlato en los desplazamientos. Un $48 \%$ de ellos en día laborable tiene su origen en el municipio de Palma, seguidos de los municipios más poblados, como Calvià (5,5\%), Manacor (5,5\%), Inca (3,6\%), Llucmajor (3,5\%) y Marratxí (3,4\%). 


\section{Metodología de análisis}

El punto de partida del análisis de los viajes de proximidad ha sido la Encuesta de Movilidad en día laborable de Mallorca 2009/2010. Dicha encuesta, encargada por el Consorcio de Transportes de Mallorca, dependiente del Gobierno Balear, ${ }^{2}$ fue realizada a través de entrevistas telefónicas (sistema CATI). Se obtuvieron 13.905 entrevistas de personas mayores de 14 años, estratificadas en función de la edad, género y municipio de residencia, con dos oleadas realizadas entre los meses de abril y junio 2009, para el municipio de Palma, y entre noviembre 2009 y febrero 2010 para el resto de la Isla. Según los datos técnicos de la propia encuesta, el margen de error para los resultados globales es de $+/-1,04 \%$ en los casos de máxima indeterminación ( $\mathrm{p}=\mathrm{q})$, para un intervalo de confianza del 95,5\%. En el cuestionario se incluyeron preguntas sobre el número de viajes realizados el día anterior, su origen y destino, el medio de transporte utilizado, la hora de realización y la duración (en minutos) del viaje, el motivo y otros datos relativos al perfil sociodemográfico del entrevistado. El resultado de la encuesta es un cálculo del número de movimientos producidos en un día laborable en toda la Isla, desglosados por orígenes y destinos, para un total de 83 zonas.

Partiendo de tales datos, en este estudio se ha individualizado y analizado los correspondientes a los viajes de proximidad. En este caso, se ha considerado como tales los movimientos dentro de una misma zona, con una duración máxima de 10 minutos, sea cual sea el modo de desplazamiento. Las zonas de la encuesta representan áreas más o menos homogéneas. En Palma coinciden con grandes agrupaciones de barrios, y en el resto de la Isla corresponden, mayoritariamente, a municipios que, en algunos casos, incluyen niveles inframunicipales, como zonas turísticas o núcleos secundarios de relativo peso (figura 2).

Siguiendo con este criterio, se ha trabajado con una base de datos de 14.262 registros pertenecientes a desplazamientos de proximidad. Aplicando los factores de expansión de la propia encuesta, equivalen a 687.003 viajes realizados en día laborable en toda la Isla. El análisis ha permitido, en primer lugar, conocer el motivo, modo, duración y perfil sociodemográfico de los viajes de proximidad, así como el peso respecto del conjunto de movimientos y su distribución territorial. En segundo lugar, y a partir de un análisis clúster mediante spss, se ha obtenido una agrupación de los registros. Se ha optado por utilizar el método de agrupación no jerárquico k-medias, adecuado cuando se trabaja con una gran cantidad de información (Pérez López, 2001). De la base de datos se ha seleccionado un total de cinco grupos de variables: género, modo de desplazamiento, motivo, situación laboral y edad. Al ser la mayor parte de ellas variables categóricas, las hemos transformado en variables dicotómicas. De este modo, se ha incluido un total de 43 variables en el análisis clúster. Después de varios análisis exploratorios, se limitó a cinco el número de conglomerados resultantes, número suficiente para conocer las agrupaciones a partir de las categorías definidas. De hecho, las pruebas realizadas con cuatro, seis u ocho grupos no han mejorado los resultados de la agrupación en cinco, debido a que el perfil sociodemográfico y el 
género, combinados, constituyen los dos criterios de mayor peso en la agrupación, que se van repitiendo en la conformación de cada uno de los grupos.

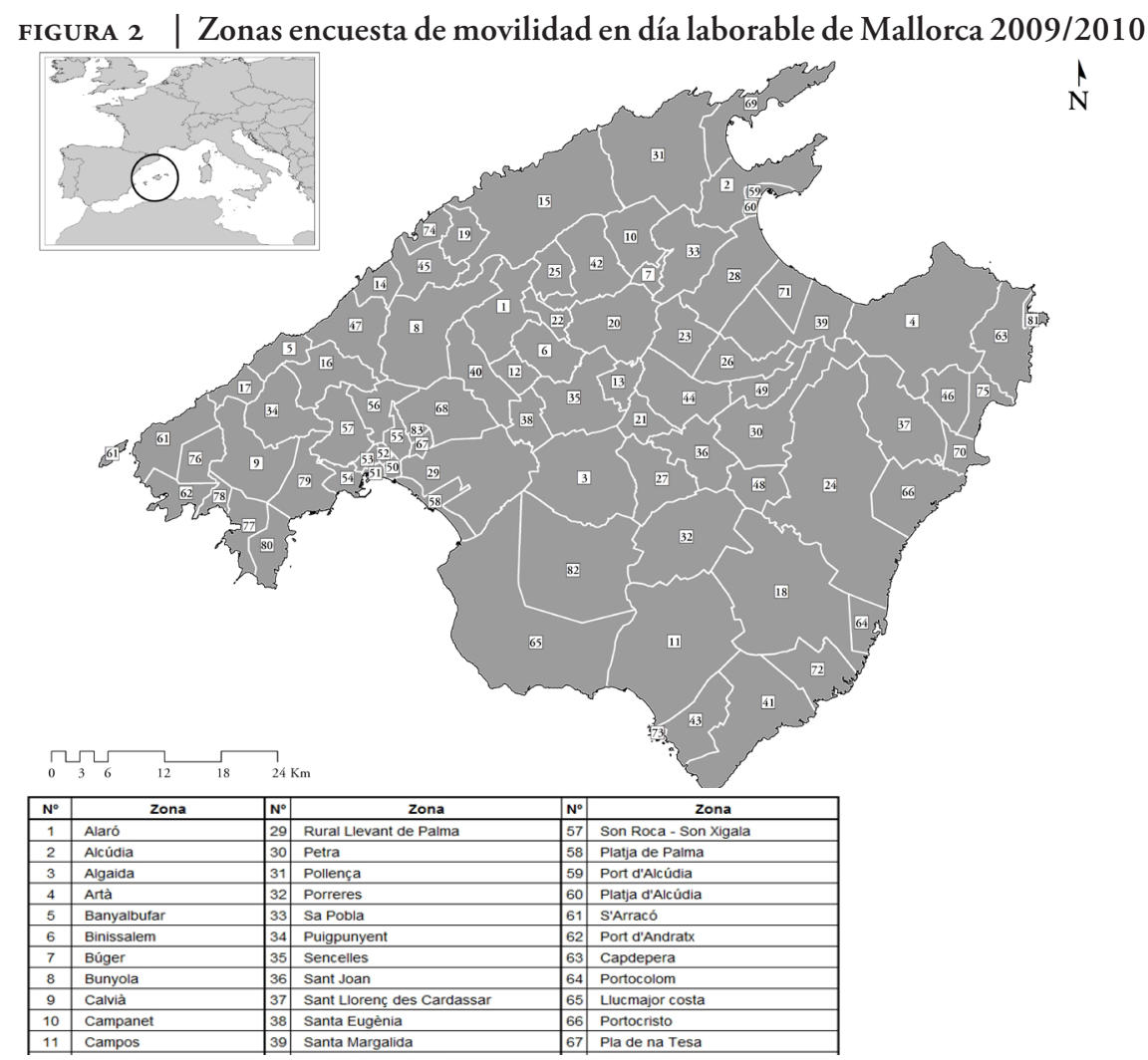

FUENTE ELABORACIÓN PROPIA

El análisis ha supuesto la determinación por iteración de los centroides de cada grupo, con un total de siete iteraciones, y la asignación de cada registro a uno de los cinco grupos resultantes, utilizando la distancia euclídea al cuadrado. La tabla 1 muestra la asignación de los registros a cada grupo resultante del proceso de cálculo.

TABLA I | Análisis clúster. Conglomerados y distribución de casos (viajes)

\begin{tabular}{|l|l|c|c|}
\cline { 3 - 4 } \multicolumn{2}{c|}{} & NO PONDERADOS & PONDERADOS \\
\hline \multirow{4}{*}{ Clúster } & 1 & 5.528 & 275.828 \\
\cline { 2 - 4 } & 2 & 2.519 & 129.056 \\
\cline { 2 - 4 } & 3 & 2.764 & 117.902 \\
\cline { 2 - 4 } & 4 & 1.737 & 94.525 \\
\cline { 2 - 4 } & 5 & 1.714 & 69.643 \\
\hline Válido & 14.262 & 687.003 \\
\hline Perdidos & 0 & 0 \\
\hline
\end{tabular}

FUENTE ELABORACIÓN PROPIA 
Por último, se ha representado cartográficamente el peso de cada uno de los conglomerados resultantes en cada zona de análisis, con la finalidad de observar las variaciones territoriales más destacadas.

\section{Resultados}

\section{Caracterización general de los desplazamientos de proximidad}

En la isla de Mallorca se realiza, en día laborable, un total de 2.281.965 desplazamientos. De ellos, 687.003 (30,1\%) corresponden a viajes de proximidad. Un primer dato interesante es el que ofrece la distribución modal comparada entre los viajes de proximidad y el total de viajes (figura 3). El uso del automóvil privado representa un $55,9 \%$ en el total de viajes, mientras que en los viajes de proximidad este peso disminuye hasta el $42,7 \%$. Por el contrario, los desplazamientos a pie alcanzan el $54 \%$ en los viajes de proximidad, mientras que en el total representan solo el 35,8\%. Destaca el poco peso de los viajes en bicicleta, con apenas $2 \%$ de los movimientos de proximidad (1,3\% en el conjunto). Esta circunstancia refleja el poco interés, con indicios de cambio reciente, por el uso de la bicicleta como modo de desplazamiento cotidiano en Mallorca, tanto en Palma como en otras ciudades y pueblos.

También es preciso destacar el diferente peso de los desplazamientos en transporte público y colectivo. Mientras que para el conjunto de viajes estos suponen el 6,7\%, en el ámbito de la proximidad su peso es muy bajo, pues únicamente alcanzan el 1,2\% de los movimientos.

\section{FIGURA 3 | Reparto modal. Viajes de proximidad vs total de viajes}

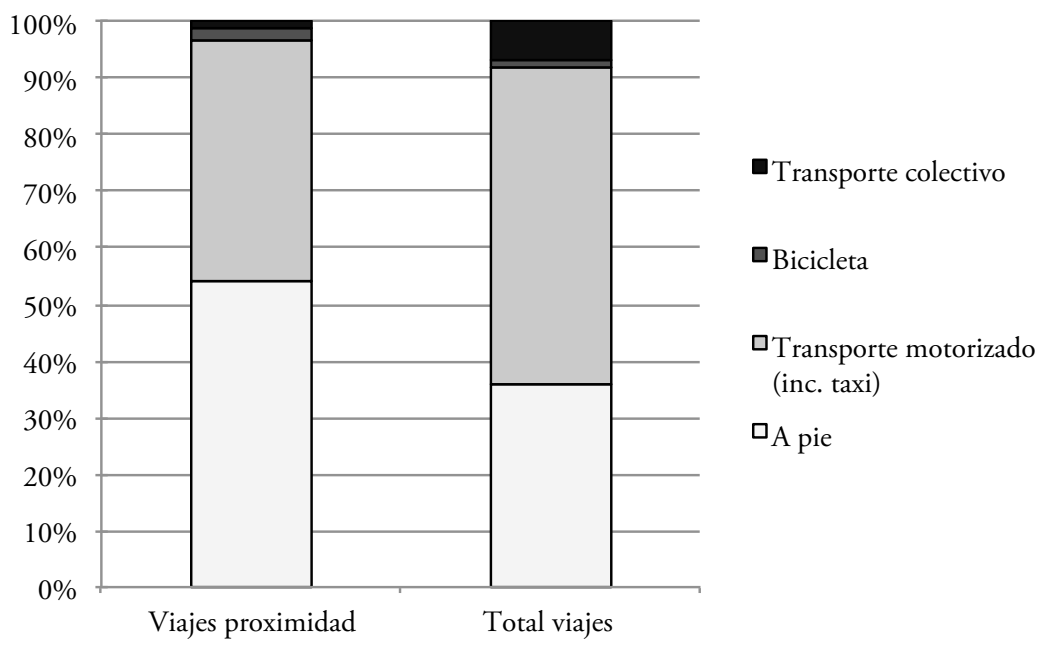

FUENTE ELABORACIÓN PROPIA 
En lo referente a los motivos del desplazamiento, las diferencias entre los viajes de proximidad y el conjunto de desplazamientos se perciben en el mayor peso de la movilidad no obligada en los primeros (40,6\% vs 36,6\%). La movilidad obligada alcanza valores relativamente bajos, tanto en los desplazamientos de proximidad $(13,3 \%)$ como en el total $(17,2 \%)$. Como se verá, ello es consecuencia de los perfiles sociodemográficos y también de las diferencias, en cuanto a localización, entre residencia y lugar de trabajo.

Si se analiza con más detalle los motivos de desplazamiento por movilidad no obligada, se observa cómo en el marco de los viajes de proximidad destacan las "compras cotidianas" (12,3\%), "acompañar personas" (9,3\%), y "ocio, diversión, espectáculos” (6,3\%), con porcentajes siempre superiores a sus equivalentes en el total de desplazamientos $(7,1 \%, 6,4 \%$ y $5,2 \%$, respectivamente). Por contra, en el ámbito de la movilidad obligada, el motivo "trabajo" es relativamente inferior en el caso de los viajes de proximidad (10,8\%) al que tiene en el total de movimientos $(13,8 \%)$, al igual que el motivo "estudios" (2,5\% vs $3,4 \%)$.

A su vez, los desplazamientos de proximidad presentan una distribución horaria diferente a la del total de viajes (figura 4), sobre todo en la hora punta de la mañana, que se sitúa entre las 10 y las 11 horas, para los primeros, cuando en el total de movimientos se adelanta dos horas, entre las 8 y las 9 horas. Por la tarde, el pico de las 15 horas para el total de viajes no se significa en los de proximidad, mientras que la hora punta de las 17 horas aparece en ambos casos.

FIGURA 4 Distribución horaria de los viajes (\%). Viajes de proximidad vs total de viajes

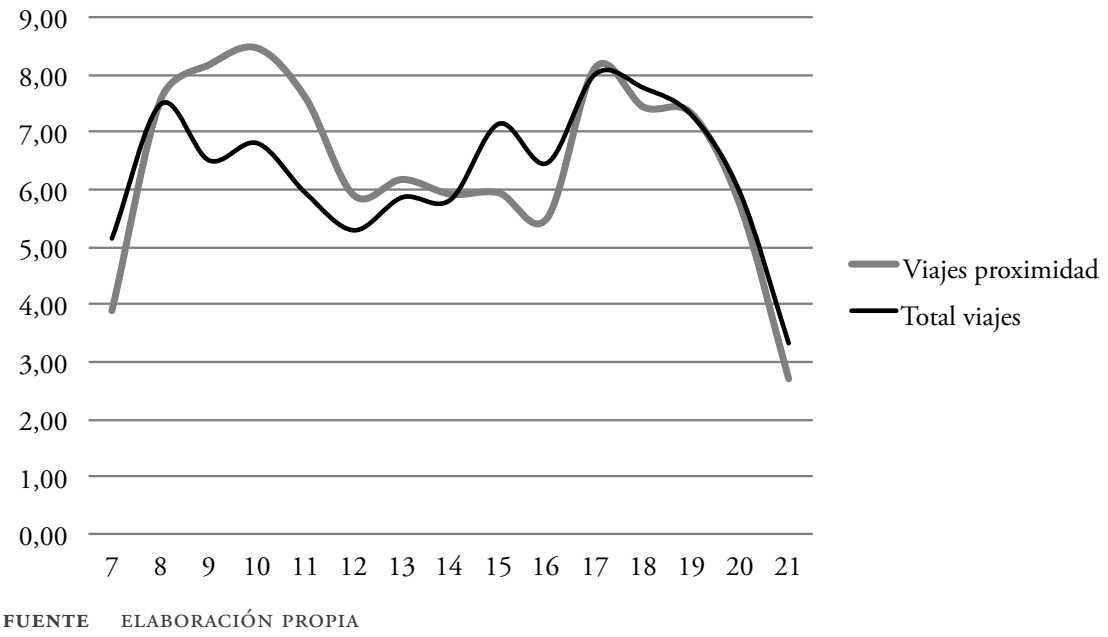

En el análisis de los perfiles sociodemográficos se observa cómo los desplazamientos de proximidad se realizan en un $44 \%$ por personas activas ocupadas. Los que generan las personas desocupadas alcanzan el 19\% del total, mientras que los jubilados o pensionistas representan el $17 \%$, seguido de las personas que realizan un trabajo doméstico 
no remunerado (10\%) y por los estudiantes (8\%). Esta distribución es bastante similar a la del total de desplazamientos, pero aun así se observan algunas diferencias destacables. Los viajes de proximidad cuentan con una menor proporción de personas activas ocupadas que el conjunto de viajes (43,9\% vs 47,7\%) y, en paralelo, son superiores las personas activas en paro (18,7\% vs $15,7 \%)$, los jubilados / pensionistas ( $17,1 \%$ vs $16,4 \%)$ y los que se dedican al trabajo doméstico no remunerado ( $9,5 \%$ vs $8,1 \%)$. En cuanto al género, y aunque esta sea una variable utilizada para la segmentación de la encuesta, destaca un ligero incremento de la proporción de población femenina en los viajes de proximidad $(53,3 \%)$ respecto del total de viajes $(50,1 \%)$.

\section{El análisis de conglomerados}

Los grupos resultantes del análisis clúster aglutinan los desplazamientos de proximidad a partir de los perfiles predominantes en cada grupo. El análisis clasifica cada uno de los registros de la base de datos en función de la distancia a cada uno de los centroides de cada variable, registros que, a su vez, representan el valor medio de cada variable en cada uno de los conglomerados. Al convertir las variables categóricas iniciales en variables dicotómicas, las que cuentan con una media más cercana a 1 son las que caracterizarán de forma más representativa el perfil propio de cada uno de los conglomerados.

Partiendo de los valores resultantes, incluidos en la tabla 1, se han identificado cinco perfiles principales que tipifican cada uno de los conglomerados:

- Grupo 1: desplazamientos correspondientes a personas activas ocupadas, con edades comprendidas entre los 34 y los 45 años, que utilizan como modo prioritario el automóvil como conductor. En este caso, los valores medios más cercanos a 1 son los que corresponden a las variables "población activa ocupada" $(0,98)$, edad entre 30 y 44 ańos $(0,58)$, género masculino $(0,53)$ y modo de desplazamiento "coche como conductor" $(0,50)$. Este es el grupo en el que se incluye el mayor número de registros, hasta un 40,2\% de los casos analizados.

- Grupo 2: desplazamientos correspondientes a personas activas en paro, de edades comprendidas entre 30 y 44 ańos, modo principal "a pie" y motivos destacados "regreso a domicilio" y "acompañar otras personas”. En este grupo los valores medios más cercanos a 1 se encuentran en las variables "personas activas en paro" $(1,00)$, modo "a pie" $(0,56)$, edad entre 30 y $44(0,49)$, motivo "regreso a domicilio" y género masculino $(0,46)$. Aunque los motivos del desplazamiento aparecen con valores más bajos, los más importantes corresponden a "regreso a domicilio" $(0,46)$ y "acompañar otras personas" $(0,17)$. A este grupo se ha asignado el 18,8\% de los casos.

- Grupo 3: corresponde a los desplazamientos de personas jubiladas, modo principal "a pie" y motivos principales "regreso a domicilio" y "compras cotidianas". En este caso, los valores más próximos a 1 son los de las variables "jubilado I pensionista” $(0,98)$, modo "a pie” $(0,76)$, edad “65 y más” $(0,73)$, género masculino $(0,49)$, y motivos del desplazamiento "regreso a domicilio" $(0,47)$ y "compras cotidianas" $(0,21)$. En esta agrupación se ha incluido el 17,2\% de los casos. 
- Grupo 4: desplazamientos de jóvenes estudiantes, modo principal “a pie” y motivo principal "estudios". En este grupo los valores medios más cercanos a 1 son los correspondientes a los grupos de edad de entre 14 y 29 años $(0,99)$, modo "a pie" $(0,68)$, perfil sociodemográfico "estudiante" $(0,61)$ y género masculino $(0,58)$. En este grupo se ha incluido el $13,8 \%$ de los registros.

- Grupo 5: este último grupo se caracteriza por desplazamientos realizados principalmente por mujeres, de perfil laboral "tareas del hogar", modo "a pie", grupos de edad entre 45 y 64 años y de 30 a 44 años, siendo los motivos principales “compras cotidianas" y "acompañar personas". El que la variable "hombre" tenga valores cercanos a 0 indica que el género femenino es mayoritario en este grupo. A este conglomerado se ha asignado el 10,1\% de los casos.

\section{Discusión}

Carme Miralles-Guasch y Àngel Cebollada analizan los elementos que caracterizan la movilidad cotidiana en la época posmoderna (Miralles-Guasch, 2009), cuyos principales rasgos son reflejo de los desplazamientos vinculados al commuting (relación diaria y cotidiana entre el lugar de residencia y el lugar de trabajo), y a la vez de las distintas y a veces ocultas realidades sociales de la movilidad, que pocas veces se convierten en protagonistas a la hora de planificar el territorio o el transporte. Tal y como explican los autores en referencia, estas realidades ocultas pertenecen a los desocupados, a las mujeres, a los jubilados, todos los cuales, si bien suelen permanecer en un plano secundario en muchos de los análisis contemporáneos, desempeñan un papel fundamental en aspectos como el cuidado de los hijos o el apoyo a los jóvenes desocupados y en situaciones sociales complejas. Tal es el "rol" que socialmente se ha asignado a las mujeres, parados y mayores pensionistas, realidades que son a la vez reflejo y consecuencia del alejamiento paulatino de las esferas de lo que David Harvey denomina, tal y como citan los autores indicados, "espacios productivos" y "espacios reproductivos". Este alejamiento de dos esferas esenciales para el desarrollo social provoca una mayor necesidad de desplazamientos, por una parte; y por otra, una diferencia en términos sociales y de género entre los modos de transporte, las distancias recorridas y los motivos de dichos desplazamientos. En esta perspectiva, se relegan los viajes más cercanos a aquellos colectivos que o bien no necesitan desplazarse por carecer de trabajo (parados, estudiantes, pensionistas), o bien no disponen de acceso al vehículo privado; o ambas cosas a la vez.

El resultado del análisis realizado en este trabajo encaja con los razonamientos teóricos expuestos, y pone de relieve el diferente comportamiento que presentan los distintos perfiles sociales y de género en los desplazamientos de proximidad. Es decir, las pautas de movilidad, la motivación de los desplazamientos y la elección de cada uno de los modos de transporte utilizados cambian en función del género y del nivel socioeconómico. El uso diferenciado del tiempo y del espacio, y las dificultades de acceso a determinados modos de transporte en función del nivel de ingresos o incluso del género, condicionan de un modo absoluto las posibilidades y opciones a la hora de realizar un desplazamiento. Este hecho también se refleja en los desplazamientos de proximidad. 
El análisis deja patente que el factor principal de agrupación es el del perfil sociodemográfico. Este, a su vez, refleja los diferentes patrones de desplazamiento de cada grupo en función de la distribución temporal de la actividad cotidiana y de los roles que cada uno de los grupos desempeña en la sociedad actual. Un análisis en profundidad de la composición de cada uno de los conglomerados resultantes permite observar con mayor detalle las diferencias entre ellos (figuras 5, 6 y 7). Los perfiles ocupacionales se reparten de forma separada entre los grupos. En el grupo 1 predominan los trabajadores en activo $(98,4 \%)$; en el 2 , las personas activas en paro (99,5\%); en el 3, los jubilados y pensionistas (98,3\%); en el grupo 4 aparecen los perfiles de estudiantes $(60,9 \%)$ como los mejor representados; y en el 5, las personas que realizan tareas domésticas no remuneradas (93,7\%). Los matices de género aparecen sobre todo en el grupo 5, compuesto casi completamente por mujeres $(95,9 \%)$, mientras que en el resto el reparto es más o menos equitativo. En el caso de la distribución por edades, en el grupo 4 están mejor representadas las edades jóvenes (99,3\% corresponde a personas entre 14 y 29 años), mientras en el grupo 3 predomina el perfil de edades de 65 años y más $(72,8 \%)$.

\section{FIgURA 5 | Perfil laboral (\%) por conglomerados}

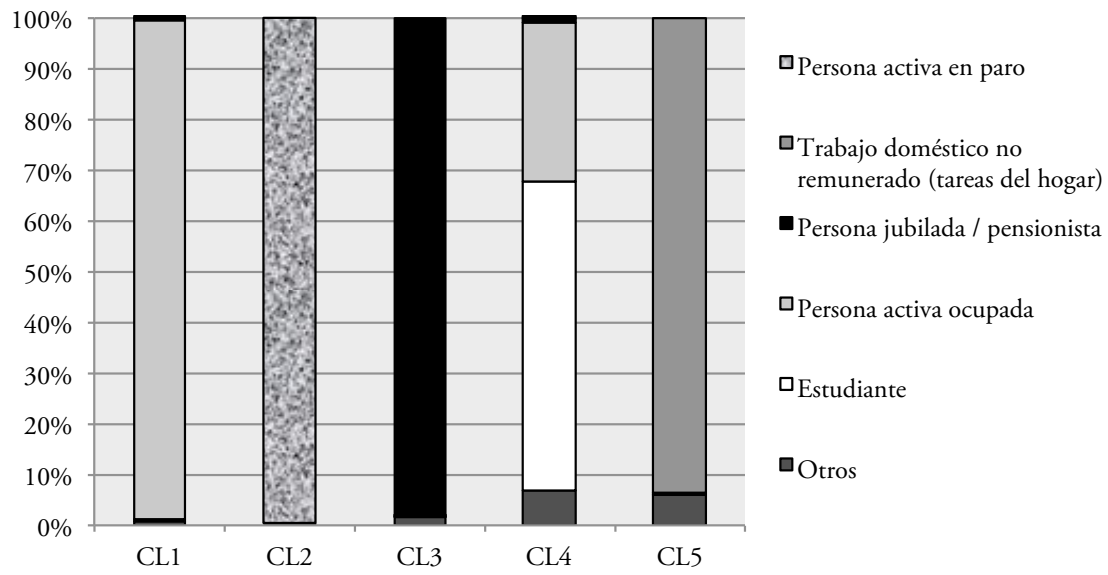

FUENTE ELABORACIÓN PROPIA 
FIGURA 6 | Distribución por género (\%) por conglomerados

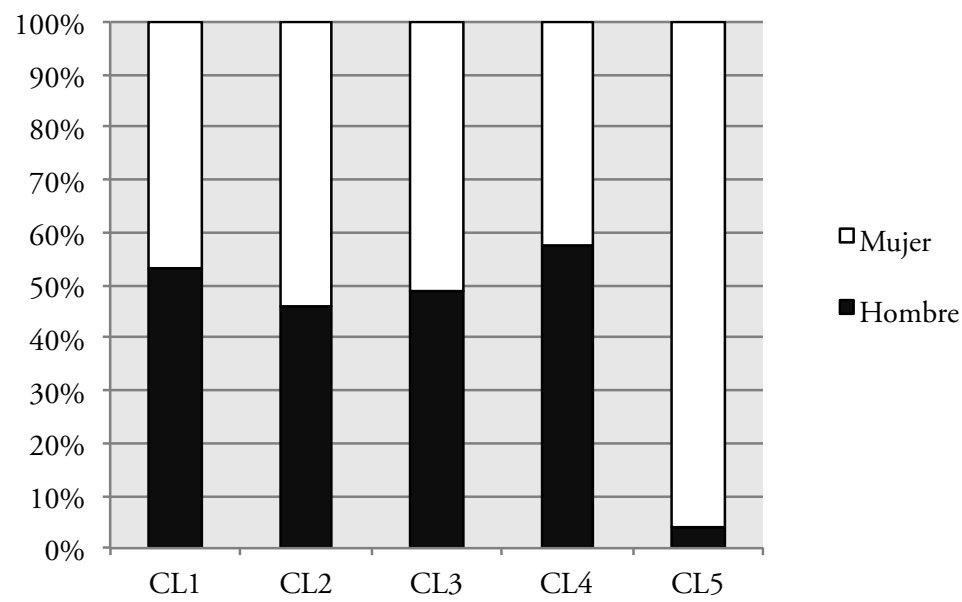

FUENTE ELABORACIÓN PROPIA

FIGURA 7 | Distribución por edades (\%) por conglomerados

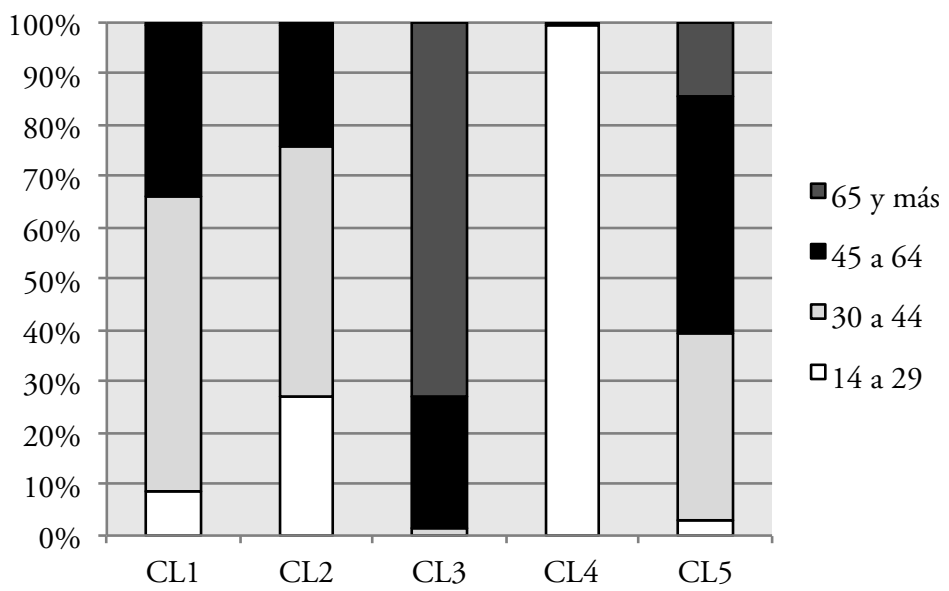

FUENTE ELABORACIÓN PROPIA

Espacio-tiempo, género y equidad social reflejados en los desplazamientos de proximidad

En el análisis expuesto aparecen claramente dos tipos de actores ante los desplazamientos de proximidad: los trabajadores en activo y el resto de grupos, que representan parados, jóvenes estudiantes, jubilados y amas de casa. La principal diferencia entre ellos se da en el reparto modal de sus desplazamientos (figura 8). En el desglose por grupos, los movimientos a pie y en bicicleta son más importantes en los perfiles 
de los estudiantes (grupo 4, con 70,7\%); pensionistas (grupo 3, con 77,5\%) y amas de casa (grupo 5, con 65,6\%). En el caso de las personas desocupadas, el porcentaje es menor y, aun así, representa el $57 \%$ del total de los viajes. Por el contrario, los trabajadores en activo son los que se desplazan mayoritariamente en transporte privado / motorizado, modo que alcanza hasta el 54,7\% de los desplazamientos. El uso del automóvil es, pues, todavía mayoritario en el caso de los trabajadores en activo, aun cuando sea para realizar desplazamientos que podrían ser sustituidos por otro modo (transporte colectivo o modos no motorizados) por su corta duración.

\section{FIGURA 8 Reparto modal por conglomerados}

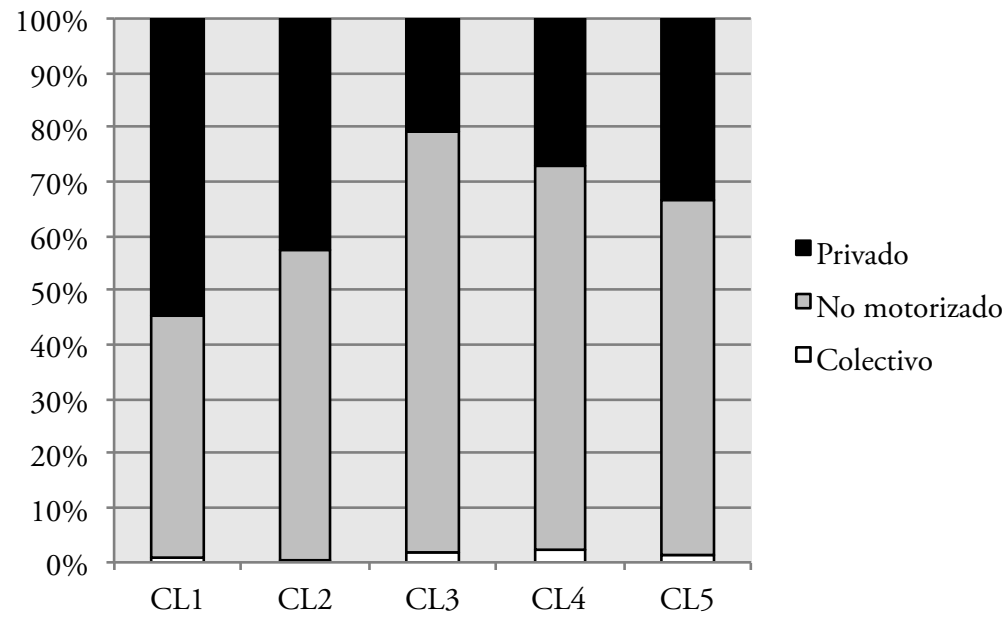

FUENTE ELABORACIÓN PROPIA

En los comportamientos cotidianos y, por ende, en la movilidad de proximidad, se manifiestan de forma evidente las diferencias en el uso entre espacio y tiempo tanto desde la perspectiva de género como desde la perspectiva social. La atribución de diferencias de género en los diferentes modos de transporte, y en la diferente organización del tiempo en nuestra vida diaria, se plasma en lo que Doreen Massey (1994) denomina "espacios sesgados desde el punto de vista de género", gendered places.

Es decir, la manera como mujeres y hombres leen el espacio y el territorio, o bien la diferente manera en que la organización espacial y temporal establecida por la sociedad genera diferencias de género, se manifiesta también en los desplazamientos de proximidad. Mujeres que trabajan en casa mayoritariamente se desplazan a pie, en comparación con trabajadores en activo que lo hacen en vehículo privado, y presentan también diferentes pautas horarias de desplazamiento -la dimensión temporal-, en función de las necesidades de un rígido horario de trabajo o de la organización basada en las necesidades de las tareas del hogar (figura 9). Aparecen a la vez dos pautas diferentes vinculadas a las características laborales: la de los estudiantes y trabajadores ocupados, con mayor movilidad a primera hora de la mańana y a primera hora de la tarde; y la de los desocupados, jubilados y amas de casa, cuyas 
horas punta se centran entre las 9 y las 12 de la mañana y a última hora de la tarde. Cabe señalar, por ejemplo, el hecho de que el $18 \%$ de los desplazamientos de las personas jubiladas, y el $14 \%$ de los de las amas de casa, se realizan a las 10 de la mañana, con gran diferencia sobre el resto de franjas horarias. Las personas desocupadas (grupo 2) presentan su máxima movilidad (11,5\%) a las 9 de la mañana, hecho que se puede relacionar claramente con desplazamientos para acompañar a otras personas, como es llevar a los hijos a la escuela.

\section{FIGURA 9 Distribución horaria de los desplazamientos por conglomerados (\% de desplazamientos en cada franja horaria)}

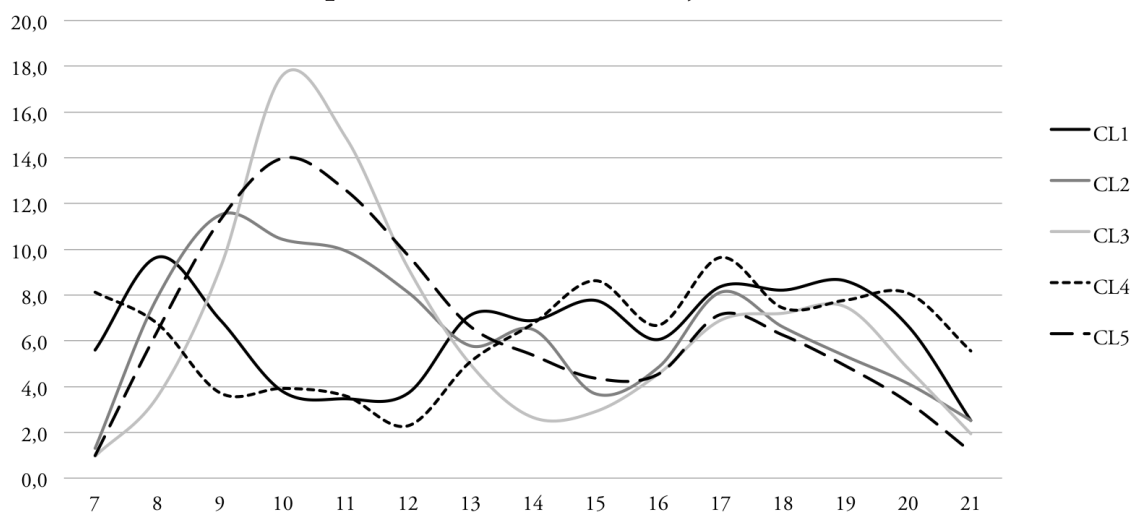

FUENTE ELABORACIÓN PROPIA

La distribución de los diferentes motivos de desplazamiento es, pues, otro de los indicadores de las diferencias socioespaciales existentes (figura 10). Si se exceptúa el motivo "regreso a domicilio", aparece que los motivos vinculados con la movilidad obligada tienen un mayor peso en los grupos de estudiantes y trabajadores en activo, en los que superan el 20\% de los movimientos (24,2\% y 25,6\%, respectivamente). En el resto de grupos, los viajes se vinculan a "médico, ocio y otros" (27,6\% en los grupos 3 y 5; 21,1\% en el grupo 4); “acompañar a otras personas”, que alcanza un máximo de $17 \%$ en el grupo 2 ; y "compras", con porcentajes del $22 \%$ en los grupos de jubilados y amas de casa. 
FIGURA Io | Motivos principales del desplazamiento (\%) por conglomerados

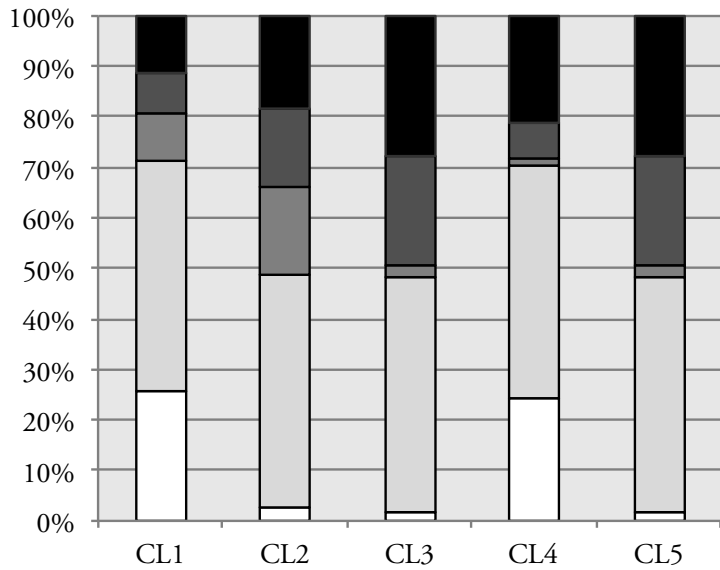

- Médico, ocio y otros

- Compras

$\square$ Acompañar personas

口Domicilio

$\square$ Obligada (estudios y trabajo)

FUENTE ELABORACIÓN PROPIA

La distribución espacial. La consolidación de la Isla como espacio metropolitano Una primera aproximación a la distribución espacial de los viajes de proximidad, en este caso en cifras absolutas, pone de manifiesto claramente la vinculación entre dichos traslados y el peso que cada municipio ostenta en el conjunto insular, en términos demográficos y territoriales. El mapa representado en la figura 11 permite entrever un primer nivel de zonas con más de 20.000 movimientos de proximidad, que coinciden con los municipios estructuradores del territorio mallorquín: Manacor, Inca, Llucmajor y varias zonas incluidas en el municipio de Palma.

\section{FIGURA I I | Distribución territorial de los viajes de proximidad por zonas}



FUENTE ELABORACIÓN PROPIA 
Sin embargo, en un segundo nivel, con valores entre 10.000 y 20.000 viajes diarios, aparecen algunos municipios en los que el peso demográfico no sería el único factor explicativo, sino que aflorarían cuestiones relacionadas con otras dinámicas, de carácter más comarcal. Es el caso de las zonas ubicadas en la zona noroeste, como Pollença, Alcúdia, Sa Pobla y Muro; o el caso singular de la zona de Sóller, que presenta rasgos diferenciados por su tradicional aislamiento del resto de la Isla.

Tres áreas destacan en un tercer nivel de movimientos: la zona de la península de levante, en el noreste insular (Artà, Capdepera); las zonas del sur y sureste (Felanitx, Campos, Santanyí); y las áreas costeras de Calvià y Andratx, en el noroeste de Mallorca. Con valores inferiores a los 5000 desplazamientos se encuentra buena parte del llano central de la Isla, el resto de los municipios del noroeste insular y los que envuelven la corona metropolitana de Palma.

FIGURA I 2 Distribución territorial del peso de los viajes de proximidad en el conjunto de viajes

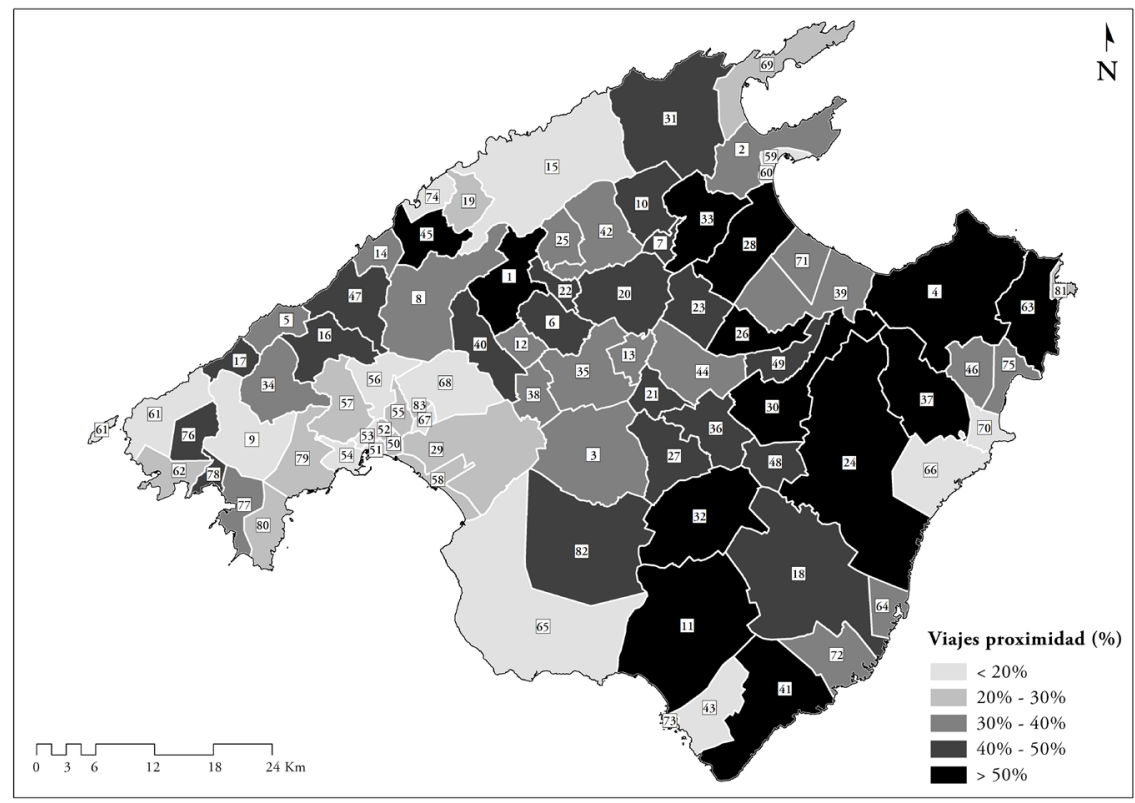

FUENTE ELABORACIÓN PROPIA

Un análisis con valores relativos de los viajes de proximidad a través del porcentaje que representan respecto del total de desplazamientos (figura 12) ofrece un mayor valor explicativo de este tipo de movimientos. Aparece nítidamente el efecto "distancia al área metropolitana de Palma”, que refleja un incremento del porcentaje de viajes de proximidad cuanto mayor es la distancia a la capital insular. Las zonas ubicadas en la comarca de levante, y algunos municipios del sur y sureste, con Manacor como núcleo aglutinador, así como algunos casos de la bahía del noreste (Muro y Sa Pobla, por ejemplo), explicitan claramente este efecto, con valores superiores 
al 50\% en este tipo de viajes. De nuevo el municipio de Sóller se singulariza en el norte, junto con Alaró, alejados de los circuitos de vías rápidas de comunicación con Palma y de transportes colectivos eficientes y competitivos.

En el lado opuesto se encuentran las áreas pertenecientes a la corona metropolitana, con porcentajes de viajes de proximidad inferiores al 30\%, así como los barrios centrales de Palma. Ello explicita los bajos índices de autocontención (cociente entre los desplazamientos con origen y destino en el barrio de residencia y el total de desplazamientos realizados por los residentes en el barrio), entre un $22 \%$ y un $28 \%$ de media y, con ello, el predominio de una movilidad cotidiana de carácter pendular, básicamente laboral, fuera de la zona de residencia.

Por último, el análisis de la distribución territorial del peso de cada uno de los conglomerados en cada zona indica cómo, a nivel general, las características descritas para el conjunto de la Isla se mantienen, aunque con algunos matices. El grupo de población activa ocupada (grupo 1), lógicamente, es también el mayoritario en la práctica totalidad de zonas. El valor porcentual oscila entre el $28 \%$ y el $60 \%$, con patrones espaciales muy repartidos. Por lo que se refiere al resto de grupos, el de las mujeres dedicadas a tareas domésticas (grupo 5) no se singulariza como mayoritario en ninguna de las zonas. El grupo 2, de personas desocupadas, presenta una mayor relevancia en áreas litorales vinculadas a espacios turísticos, y ello se explica porque la encuesta se realizó en los meses de invierno, cuando la actividad turística es muy baja y los niveles de paro elevados. El grupo de pensionistas y jubilados es mayoritario en tres zonas, seguramente debido a las propias dinámicas demográficas.

\section{Conclusiones}

La evolución territorial de la isla de Mallorca hacia un espacio metropolitano ha conllevado el incremento, muy por encima de otras regiones vecinas, del peso del vehículo privado, síntoma de un alejamiento paulatino de los espacios residenciales y los espacios laborales, y a la vez de un mayor prestigio del vehículo privado como modo de transporte, favorecido por un débil desarrollo del sistema de transporte público isleńo. Por el contrario, los desplazamientos de proximidad analizados en detalle presentan un mayor peso de los desplazamientos no motorizados, hecho que corrobora que este tipo de desplazamientos favorece una movilidad más sostenible y cercana. No obstante, estos desplazamientos no suponen más que el 30\% del total de viajes diarios.

La caracterización basada en el análisis clúster de los desplazamientos de proximidad realizada permite afinar y matizar de forma importante este análisis. La agrupación por conglomerados ha hecho aflorar la situación laboral y el perfil demográfico como factores principales de agrupación. Estos perfiles, analizados separadamente, permiten identificar cada uno de estos colectivos y sus hábitos cotidianos. Y cabe constatar que el grupo que incluye un mayor número de casos (cerca del $40 \%$ ) es el que corresponde a trabajadores jóvenes en activo, que presentan un mayor peso del vehículo privado en sus desplazamientos, aun tratándose de desplazamientos de proximidad. Por tanto, la relación entre el perfil laboral y el uso 
del vehículo privado es elevada, incluso cuando no existe una gran separación física entre residencia y centro de trabajo.

Para contrastar esta afirmación, puede observarse cómo los parados, amas de casa y pensionistas son los colectivos con un mayor peso de los desplazamientos no motorizados en el ámbito de los viajes de proximidad. Por tanto, los colectivos que no dependen de un desplazamiento laboral son los que realizan un mayor número de viajes en modo no motorizado. Unas pautas horarias en los desplazamientos diferentes de los trabajadores en activo, junto con las barreras para acceder a un vehículo privado que generalmente se asocian a estos colectivos, explican también este hecho. Estos serían, pues, factores clave que darían cuenta del mayor peso de la movilidad sostenible en los desplazamientos próximos. Por tanto, los factores relacionados con la distancia o con el tipo de tejido urbano existente no son los únicos que esclarecen las diferencias, en cuanto a modos de transporte, entre viajes de proximidad y el resto de viajes.

Aun así, no cabe duda de que la tendencia social hacia la hipermovilidad responde a un proceso de mayor fragmentación de los usos del tiempo, y a la dispersión espacial de los usos del suelo. La combinación de ambos factores incrementa la necesidad de desplazarse cada vez más lejos. Ello conlleva un incremento de las desigualdades, ya que no toda la población tiene el mismo acceso al transporte privado; y supone un mayor impacto ambiental, debido a la contribución del cambio climático generada por las emisiones, los efectos de la congestión y otras externalidades que no son objeto de este trabajo y que caracterizan la mayor parte de ciudades en entornos desarrollados. En este contexto, proximidad y accesibilidad aparecen como elementos que se deben tener muy presentes en la reflexión sobre la evolución futura del modelo territorial que genera la dispersión de actividades y los procesos de ciudad difusa. Uno de los efectos inmediatos es la mayor necesidad de movimientos. Esta hipermovilidad, sobre todo por carretera, en el contexto de desplazamientos metropolitanos, aunque no exclusiva de este modo, aparece asociada a un uso más extensivo y especializado del espacio en un entorno más global. El territorio de proximidad y sus viajes permiten revertir precisamente los efectos de la dispersión. Sin embargo, en este debate es crucial prestar atención a las diferencias que se producen por motivos sociales o de género. El poder acceder a servicios y actividades a poca distancia y a través de medios no motorizados comporta mayores niveles de justicia social, más calidad de vida y menor impacto ambiental, pero no deben quedar relegados a estos desplazamientos solo los colectivos que no pueden tener otra opción de movilidad.

Tal y como se ha analizado en el presente artículo, el modelo urbano y de redes de transporte de las últimas décadas ha contribuido a separar los centros de trabajo ubicados en la ciudad de Palma del resto de la Isla, que se configura como un gran espacio residencial, uno que presenta un mayor gradiente a medida que nos alejamos de la capital, Palma, que es el mayor polo de concentración de centros de trabajo. El mayor impacto de la "metropolitanización" lo presentan los municipios más cercanos a la capital, como demuestra el menor peso de los desplazamientos de proximidad en estos ámbitos. Cabría ver si factores sociodemográficos, como menores tasas de envejecimiento o mayores tasas de actividad, explicarían también 
esta situación. Pero, a grandes rasgos, las zonas más alejadas de la ciudad, y alguna de ellas de las vías rápidas, como los pueblos de la parte central de la Isla y otros núcleos con entidad demográfica suficiente que se han mantenido siempre más autónomos de la capital, aún conservan algunas de las características de la ciudad tradicional más compacta y con mezcla de usos, que se traducen en una mayor generación de desplazamientos de proximidad.

No cabe duda de que la planificación territorial y de movilidad en la Isla carece de herramientas suficientes para apostar por un modelo más sostenible. A la vista de las conclusiones presentadas, parece razonable afirmar que, por una parte, es necesario impulsar de forma clara estrategias para favorecer la movilidad no motorizada y en transporte público de los trabajadores en activo; $y$, a la vez, generar procesos de convergencia entre espacios residenciales y espacios laborales que permitan reducir de forma clara los desplazamientos a largas distancias.

\section{Referencias bibliográficas}

Ben-Elia, E., Alexander, B., Hubers, C. \& Ettema, D. (2014). Activity fragmentation, ICT and travel: An exploratory Path Analysis of spatiotemporal interrelationships. Transportation Research Part A: Policy and Practice, 68, 56-74. doi:10.1016/j.tra.2014.03.016

Buliung, R. N. (2011). Wired people in wired places: Stories about machines and the geography of activity. Annals of the Association of American Geographers, 101(6), 1365-1381. doi: 10.1080/00045608.2011.583568

Currie, G. (2010). Quantifying spatial gaps in public transport supply based on social needs. Journal of Transport Geography, 18(1), 31-41. doi:10.1016/j.jtrangeo.2008.12.002

De Vos, J. \& Witlox, F. (2013). Transportation policy as spatial planning tool: Reducing urban sprawl by increasing travel costs and clustering infrastructure and public transportation. Journal of Transport Geography, 33, 117-125. doi:10.1016/j.jtrangeo.2013.09.014

Esteban, J. (2006). La necesaria contención de la dispersión urbana. En A. Tarroja \& R. Camagni (Eds.), Una nueva cultura del territorio (pp. 267-277). Barcelona: Diputació de Barcelona. Consorci Universitat Internacional Menénedez Pelayo de Barcelona (сиімгв) Centre Ernest Lluch.

García-Palomares, J. C. (2010). Urban sprawl and travel to work: The case of the metropolitan area of Madrid. Journal of Transport Geography, 18(2), 197-213. doi:10.1016/j. jtrangeo.2009.05.012

Indovina, F. (coord). (2007). La ciudad de baja densidad. Barcelona: Diputació de Barcelona.

Ludlow, D. (2006). Urban sprawl in Europe - The ignored challenge. doi:10.1080/02697451003740312

Marquet, O. \& Miralles-Guasch, C. (2013). La dimensión social de las dinámicas de proximidad en Barcelona. Smart Regions for a Smarter Growth Strategy: New challenges of the regional policy and potentials of cities to overcome a worldwide economic crisis, 18022. http:// www.reunionesdeestudiosregionales.org/Oviedo2013/htdocs/pdf/p740.pdf 
Massey, D. B. (1994). Politics and space / time. En Space, place and gender (pp. 249-272). Minneapolis, MN: Universisty of Minnesota Press. doi:10.1049/el:19990302. Primera versión en New Left Review, 1(196), 65-84 (nov.-dic. 1992).

Mestre Morey, M. (2014). La delimitació de l' àrea urbana de Palma de Mallorca. Una reconsideració a partir de l' aplicació del criteri nurec. Treballs de La Societat Catalana de Geografia, 76, 103-126. doi:10.2436/20.3002.01.41

Miralles-Guasch, C. (2002). Ciudad y transporte: el binomio imperfecto. Barcelona: Ariel.

Miralles-Guasch, C. (2009). Movilidad cotidiana y sostenibilidad. Una interpretación desde la Geografía Humana. Boletín de la Asociación de Geógrafos Españoles (AGE), (50), 193216. http://boletin.age-geografia.es/articulos/50/08\%20MIRALLES.pdf

Muñoz, F. (2005). La producció residencial de baixa densitat. Serie Elements de debat territorial, $\mathrm{n}^{\circ} 21$. Barcelona: Diputació de Barcelona.

Nel.lo, O. (2012). Francesco Indovina: del análisis del territorio al gobierno de la ciudad. Barcelona: Icaria.

Pérez López, C. (2001). Técnicas Estadisicas con SPSS. Editado por P. Hall. Madrid: Universidad Complutense de Madrid.

Quintana Peñuela, A. (1979). El sistema urbano de Mallorca. Palma: Ed. Moll.

Seguí Pons, J. M. (2009). Infraestructures i territori. En M. Castañer \& À. Colomer (Eds.), Jornades sobre sostenibilitat i territori. Els limits del creixement als Països Catalans. Lleida, 23 i 24 de novembre de 2007 (pp. 19-40). Lleida: Fundació Congrés de Cultura Catalana.

Travisi, C. M., Camagni, R. \& Nijkamp, P. (2010). Impacts of urban sprawl and commuting: A modelling study for Italy. Journal of Transport Geography, 18(3), 382-392. doi:10.1016/j.jtrangeo.2009.08.008

Wefering, F., Rupprecht, S., Bührmann, S., Böhler-Baedeker, S. \& Rupprecht Consult. (2013). Developing and implementing a sustainable urban mobility plan. Bruselas: European Commission, Directorate-General for Mobility and Transport. 